Journal of Environmental
Analysis and Progress
ISSN: $2525-815 \mathrm{X}$

\title{
Análise quali-quantitativa da arborização da Praça Chácara Dona Catarina em Cataguases, Minas Gerais
}

\section{Quali-quantitative analysis of the afforestation of the Square Chácara Dona Catarina in Cataguases, Minas Gerais}

\author{
Frederico Dalton ${ }^{\mathrm{a}}$, Thiago Pinheiro Nunes ${ }^{\mathrm{b}}$, Angeline Martini ${ }^{\mathrm{a}}$
}

${ }^{a}$ Universidade Federal de Viçosa-UFV, Departamento de Engenharia Florestal. Avenida Peter Henry Rolfs, s/n, Campus Universitário, Viçosa, Minas Gerais, Brasil. CEP: 36570-900. E-mail: dalton.s.frederico@gmail.com (autor correspondente), martini@ufv.br

b Prefeitura Municipal de Cataguases, Secretaria de Agricultura e Meio Ambiente. Rua Gama Cerqueira, 70, Vila Domingos Lopes, Cataguases, Minas Gerais, Brasil. CEP: 36774-009. E-mail: pinheironunes@ hotmail.com.

\begin{tabular}{l}
\hline A R T I C L E I N F O \\
\hline Recebido 27 Jul 2020 \\
Aceito 08 Abr 2021 \\
Publicado 08 Out 2021
\end{tabular}

Publicado 08 Out 2021

\begin{abstract}
A B S T R A C T
The Chácara Dona Catarina Square is considered one of the main urban green areas in the city of Cataguases, Minas Gerais. It has an important participation in the historical and social context of the city. The study aimed to carry out a qualitative and quantitative assessment of afforestation, aiming to provide detailed information on the condition of the species to collaborate with the management and maintenance of this green area. All the tree and palm trees in the square were quantified and botanically identified. Analyzes of plant health and potential targets in a possible case of a fall were carried out for each individual, in addition to the measurement of the Chest Height Circumference (CHC) and the classification of the specimens in diameter classes. In total, 89 individuals of 21 species were found, distributed in nine botanical families and 19 genera, and most of the trees were classified as native to the region. The afforestation of the place provides a good area for leisure and social interaction of the regulars. However, it is necessary to have a systematic assessment system of the trees since some individuals presented phytosanitary problems.
\end{abstract}

Keywords: Diagnosis of afforestation, urban squares, phytosociology.

\section{R E S U M O}

A Praça Chácara Dona Catarina é uma das principais áreas verdes urbanas da cidade de Cataguases, Minas Gerais e tem importante participação no contexto histórico social do município. O estudo objetivou realizar uma avaliação quali-quantitativa da arborização da praça, visando fornecer informações detalhadas sobre a condição das espécies, de modo a colaborar com a gestão e manutenção desta área verde. Todos os indivíduos arbóreos e palmeiras presentes na praça foram quantificados e identificados botanicamente. Análises da fitossanidade e dos alvos potenciais em um possível caso de queda, foram realizadas para cada indivíduo, além da medição da Circunferência à Altura do Peito (CAP) e a classificação dos exemplares em classes de diâmetro. No total, foram encontrados 89 indivíduos de 21 espécies, distribuídos em nove famílias botânicas e 19 gêneros, com a maior parte das árvores classificada como nativas da região. A arborização do local proporciona uma boa área para lazer e convívio social dos frequentadores, porém, é necessário que se tenha um sistema de avaliação sistemático das árvores, visto que alguns indivíduos apresentam problemas fitossanitários.

Palavras-Chave: Diagnóstico da arborização, praças urbanas, fitossociologia.

\section{Introdução}

As áreas verdes urbanas, especialmente as praças, apresentam-se como um importante espaço público para a população que as utilizam, sobretudo no Brasil, onde as praças foram inseridas em seu contexto histórico como pontos de encontro 
e de comércio, originando o desenvolvimento de diversas cidades (Benini \& Martin, 2010). As praças são locais que contribuem diretamente para o aumento do convívio social de uma cidade, principalmente por se tratar de um ambiente que carrega características culturais do local, e, além de valorizar a paisagem, podem ser utilizadas como ferramentas de atividades políticas, administrativas, culturais, religiosas, econômicas e de lazer (Silva et al., 2019).

As praças também atuam para melhoria da qualidade de vida das pessoas, uma vez que amenizam as consequências negativas do expansionismo urbano, como o desconforto térmico, a monotonicidade da paisagem, a piora da qualidade do ar, o aumento da poluição sonora, entre outros efeitos prejudiciais ao bem estar e à saúde humana (Romani et al., 2012). Desta forma, atuam para reverter muitos dos problemas provocados pelo avanço da urbanização, que tornam-se ainda mais agravantes quando não se leva em consideração o processo de planejamento, e a relação entre a fisionomia da cidade com as características ambientais e culturais da região (Melo \& Romanini, 2008).

A conciliação de um projeto arquitetônico com um planejamento paisagístico que conserve as características ecológicas e ambientais, proporciona melhoria na qualidade de vida da população urbana que utiliza, entre as diferentes formas possíveis, as áreas verdes das cidades (Oliveira et al., 2013). Para isso, um bom planejamento das áreas verdes deve ser realizado. O que inclui a realização de um inventário arbóreo para caracterizar a variedade de árvores e palmeiras de um local (Santos et al., 2011).

O diagnóstico de arborização em áreas específicas é fundamental para a gestão correta dos bens públicos, uma vez que o conhecimento do patrimônio arbóreo das praças permite conhecer as condições desses ambientes e detectar possíveis necessidades de manejo (Silva et al., 2019). Além disso, os inventários da arborização tornaram-se valiosas fontes de informações para nortear o desenvolvimento de pesquisas referentes aos serviços ambientais, sociais e econômicos gerados pelas árvores urbanas (Nielsen, Östberg \& Delshammar, 2014).

Todos os múltiplos serviços ecossistêmicos fornecidos pelas árvores na paisagem urbana, fazem com que o interesse pela realização de inventários da arborização seja crescente ao longo dos últimos anos, como uma resposta a maior conscientização entre os tomadores de decisão (Hubacek \& Kronenberg, 2013). Portanto, entendendo a importância da realização de inventários da arborização em praças, como uma ferramenta que possibilita um melhor gerenciamento e tomada de decisões mais assertivas sobre tais espaços públicos, o objetivo dessa pesquisa foi realizar uma avaliação qualiquantitativa das árvores presentes na Praça Chácara Dona Catarina em Cataguases, Minas Gerais.

\section{Material e Métodos}

A pesquisa foi realizada em Cataguases, considerada uma cidade histórica do estado de Minas Gerais. O município possui uma área de $491,7 \mathrm{~km}^{2}$ com a população estimada em 75.123 habitantes (IBGE, 2020), e está localizado na zona da mata mineira (Figura 1).

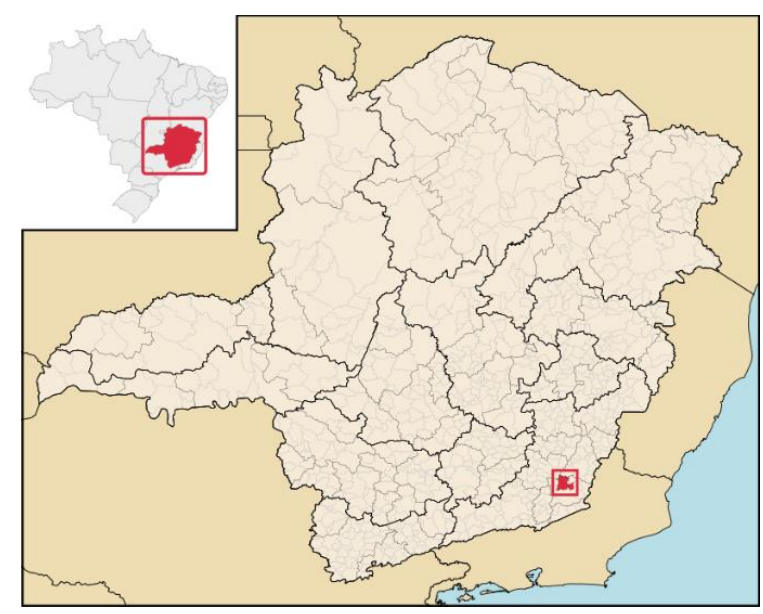

Figura 1. Localização do Município de CataguasesMG. Fonte: Abreu (2020).

A Praça Chácara Dona Catarina (Figura 2) se encontra nas coordenadas geográficas de $21^{\circ} 23^{\prime} 10^{\prime \prime}$ Sul e $42^{\circ} 41^{\prime} 30^{\prime \prime}$ Oeste.

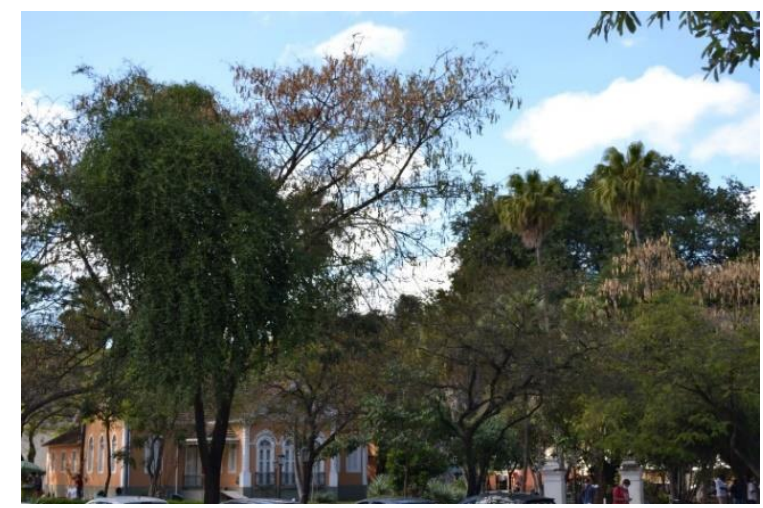

Figura 2. Imagem da Praça Chácara Dona Catarina, em Cataguases-MG. Fonte: Dalton, Nunes \& Martini (2021).

A cidade está localizada na sub-bacia do Rio Pomba, pertencente a bacia do Rio Paraíba do Sul, e a fitofisionomia florestal é característica da Floresta Estacional Semidecidual, pertencente ao bioma da Mata Atlântica. Segundo o IBGE (2020), $87,5 \%$ dos domicílios urbanos estão em vias 
públicas com arborização. O clima da região, segundo a classificação de Köppen, é do tipo Cwa, ou subtropical úmido, onde os verões são quentes e úmidos e os invernos são secos (Kottek et al., 2006). A temperatura média anual é de $24,5^{\circ} \mathrm{C}$, com máximas e mínimas de $31^{\circ} \mathrm{C}$ e $18^{\circ} \mathrm{C}$, respectivamente. O índice pluviométrico anual é de $1.564 \mathrm{~mm}$, com as chuvas concentradas no período de outubro a março (Paula et al., 2015).

Fundada pelo colonizador francês Guido Thomas Marlière, Cataguases começou a se tornar importante no polo cultural brasileiro, na década de 1920, graças, principalmente, aos trabalhos do cineasta Humberto Mauro. A referência cultural da cidade fez com que Cataguases ficasse a frente do Movimento Moderno da arquitetura nas décadas de 1940 até 1960, fazendo com que grandes nomes da arquitetura como Oscar Niemeyer, Cândido Portinari e Burle Marx deixassem suas marcas na estética arquitetônica do município (Alonso, 2012a). Devido a estas influências, o Instituto do Patrimônio Histórico e Artístico Nacional (IPHAN) decidiu pelo tombamento de prédios e áreas verdes do polígono central da cidade (Alonso, 2012b; Santos \& Lage, 2005).

Dentre estas áreas tombadas, encontra-se a Praça Chácara Dona Catarina, local adotado como objeto deste estudo, visto que o elevado fluxo de pessoas e a grande variedade arbórea desta praça é superior a qualquer outra área verde tombada pelo IPHAN no município de Cataguases. Desta forma, todas as árvores e palmeiras encontradas na praça foram quantificadas, identificadas e submetidas a avaliações qualitativas. $\mathrm{O}$ inventário da arborização foi realizado nos meses de janeiro e fevereiro de 2020 e as informações de campo foram preenchidas em uma ficha técnica elaborada especificamente para este estudo, onde foram registrados: 1) Espacialização dos indivíduos presentes na praça com base em um croqui; 2) Identificação botânica dos indivíduos: feita em campo por profissionais da área de engenharia florestal e biologia. Quando não identificadas de imediato, foram utilizadas chaves dicotômicas de identificação (Simão et al., 2017; Souza \& Lorenzi, 2007); 3) Medição da Circunferência a Altura do Peito (CAP): realizada com o uso de fita métrica. Para indivíduos bifurcados abaixo de 1,30 m (altura estabelecida como altura do peito), mensurou-se os CAP's de todos os fustes, e o CAP total foi determinado a partir de um somatório.

Visando transformar algumas características qualitativas em valores numéricos, classificou-se, em três níveis, alguns dos atributos arbóreos levantados em campo (Quadro 1).

Quadro 2. Detalhes da classificação utilizada para a análise qualitativa da arborização presente na Praça Chácara Dona Catarina, em Cataguases-MG. Fonte: Dalton, Nunes \& Martini (2021).

\begin{tabular}{|c|c|c|c|}
\hline Classificação & Nível 1 & Nível 2 & Nível 3 \\
\hline $\begin{array}{l}\text { Qualidade de } \\
\text { Sombreamento }\end{array}$ & $\begin{array}{c}\text { Folhagem densa e } \\
\text { distribuída uniformemente } \\
\text { por toda copa, } \\
\text { promovendo um } \\
\text { sombreamento adequado }\end{array}$ & $\begin{array}{l}\text { Folhagem parcialmente } \\
\text { adensada, não sendo } \\
\text { inteiramente uniforme ao } \\
\text { longo da copa, gerando } \\
\text { um sombreamento } \\
\text { intermediário no local }\end{array}$ & $\begin{array}{c}\text { Copa com folhagem rala e } \\
\text { desuniforme, produzindo } \\
\text { um sombreamento } \\
\text { inadequado }\end{array}$ \\
\hline Fitoss & $\begin{array}{c}\text { Boa, indivíduo sem } \\
\text { nenhuma presença de } \\
\text { pragas ou doenças nos } \\
\text { troncos, folhas e raízes }\end{array}$ & $\begin{array}{l}\text { Regular, leve exposição } \\
\text { do indivíduo à pragas ou } \\
\text { doenças em algumas de } \\
\text { suas estruturas }\end{array}$ & $\begin{array}{c}\text { Ruim, presença severa de } \\
\text { pragas ou doenças em } \\
\text { uma ou mais partes da } \\
\text { planta }\end{array}$ \\
\hline Alvos & $\begin{array}{l}\text { Nenhuma estrutura física } \\
\text { poderia ser atingida e não } \\
\text { há movimento de } \\
\text { pedestres no alcance da } \\
\text { árvore }\end{array}$ & $\begin{array}{c}\text { Alvos de baixo valor } \\
\text { poderiam ser atingidos, } \\
\text { como uma lixeira ou uma } \\
\text { luminária, há também um } \\
\text { baixo fluxo de pedestres } \\
\text { no local }\end{array}$ & $\begin{array}{l}\text { Objetos de alto valor } \\
\text { podem ser atingidos, } \\
\text { como veículos e } \\
\text { construções históricas, } \\
\text { além disso, há uma alta } \\
\text { circulação de pedestres no } \\
\text { entorno } \\
\end{array}$ \\
\hline Substituibilidade & $\begin{array}{c}\text { Há um espaço amplo o } \\
\text { suficiente no canteiro para } \\
\text { que o indivíduo possa ser } \\
\text { substituído }\end{array}$ & $\begin{array}{l}\text { Poderiam haver } \\
\text { dificuldades na abertura } \\
\text { de uma nova cova para } \\
\text { realizar a substituição }\end{array}$ & $\begin{array}{l}\text { Dificilmente o indivíduo } \\
\text { poderá ser substituído, } \\
\text { devido à falta de espaço } \\
\text { no canteiro em que se } \\
\text { encontra }\end{array}$ \\
\hline
\end{tabular}

Uma pesquisa bibliográfica sobre as espécies identificadas na praça também foi realizada, buscando gerar mais informações sobre os indivíduos. As variáveis abordadas nesta etapa 
foram: 1) Checagem e atualização dos nomes científicos, gêneros e famílias das espécies, realizada com a busca no site Flora do Brasil (Jardim Botânico do Rio de Janeiro, 2020); 2) Classificação quanto a origem das espécies: exóticas - ocorrência natural fora do território brasileiro; nativas do Brasil - ocorrem naturalmente no país, em um dos biomas existentes; nativas locais - ocorrência natural na Floresta Estacional Semidecidual (Jardim Botânico do Rio de Janeiro, 2020); 3) Classificação quanto ao potencial de invasão da espécie: com base na lista do Instituto Hórus foi possível identificar as espécies consideradas invasoras no Brasil; 4) Presença de frutos atrativos: identificação das espécies com fruto atrativo a fauna silvestre ou a espécie humana; 5) Persistência das folhas: as espécies foram classificadas em perenifólia, semidecídua e decídua (Aragão, 2016; Santana, Silva \& Silva, 2016).

Após a coleta de informações em campo e da pesquisa bibliográfica, os dados foram processados para obter mais algumas informações necessárias ao estudo fitossociológico da praça: 1) Medição da área total da praça Chácara Dona Catarina, usando o software Google Earth Pro; 2) Cálculo dos valores de Diâmetro à Altura do Peito (DAP) e divisão dos exemplares em centros de classe de DAP, com o uso do software Microsoft Excel; 3) Índice de Risco: associação dos valores da fitossanidade com a medida dos alvos potenciais, também realizada por meio do Microsoft Excel. Desta forma, tal somatória tem variação de 2 até 6 e, a partir desse valor, foram estabelecidas as classes de Índice de Risco e as ações de manejo correlacionadas (Tabela 1).

Tabela 1. Classificação do índice de risco e recomendação da periodicidade de reavaliação para a arborização da Praça Chácara Dona Catarina, em Cataguases-MG. Fonte: Dalton, Nunes \& Martini (2021).

Índice de Risco

$$
\begin{gathered}
\text { Risco Baixo }\left(\sum \mathrm{IR}=2 \text { ou } 3\right) \\
\text { Risco Médio }\left(\sum \mathrm{IR}=4 \text { ou } 5\right) \\
\text { Risco Alto }\left(\sum \mathrm{IR}=6\right)
\end{gathered}
$$

Periodicidade da Avaliação

$$
\begin{gathered}
2 \text { anos } \\
1 \text { ano } \\
6 \text { meses }
\end{gathered}
$$

Quanto à análise fitossociológica, foram calculados os parâmetros frequência, densidade e dominância das espécies. As Frequências Absoluta e Relativa estão associadas ao número de unidades amostrais em que uma espécie ocorre em relação ao número total de unidades amostrais. Foram calculadas pela Equação 1 e Equação 2 (MuellerDombois \& Ellenberg, 1974):

$$
\begin{aligned}
& \mathrm{FAi}=\frac{\mathrm{UAi}}{\mathrm{UAt}} \\
& \mathrm{FRi}=\left(\frac{\mathrm{FAi}}{\Sigma \mathrm{FA}}\right) * 100
\end{aligned}
$$

onde: $\mathrm{FAi}=$ Frequência Absoluta da espécie i; FRi $=$ Frequência Relativa da espécie $\mathrm{i}(\%)$; UAi $=$ Número de Unidades Amostrais com ocorrência da espécie i; UAt $=$ Número de Unidades Amostrais total.

A Densidade Absoluta e Relativa, demonstram o número de indivíduos de uma determinada espécie por área amostrada. Tais informações foram calculadas com o uso da Equação 3 e Equação 4 (Mueller-Dombois \& Ellenberg, 1974):

$$
\mathrm{DAi}=\frac{\mathrm{Ni}}{\mathrm{A}}
$$

$$
\mathrm{DRi}=\left(\frac{\mathrm{DAi}}{\Sigma \mathrm{DA}}\right) * 100
$$

onde: $\mathrm{DAi}=$ Densidade Absoluta da espécie $\mathrm{i}$ (indivíduos/ha); DRi = Densidade Relativa da espécie i (\%); $\mathrm{Ni}=$ Número de indivíduos da espécie i; A = Área total amostrada (hectares).

Além disso, a partir da coleta da Circunferência a Altura do Peito, e sua transformação para Diâmetro a Altura do Peito, calculou-se os valores de Área Basal de cada indivíduo e, consequentemente, de cada espécie (Rodrigues, 2019). O cálculo da Área Basal possibilitou a estimativa da Dominância Absoluta e Relativa, que reflete na informação sobre qual espécie ocupa maior área da unidade amostral, em termos de tamanho do tronco, considerando a Equação 5, a Equação 6 e a Equação 7 (MuellerDombois \& Ellenberg, 1974):

$$
\begin{aligned}
& \mathrm{Gj}=\frac{\left(\pi * \mathrm{DAPj}^{2}\right)}{4} \\
& \text { DOAi }=\frac{\Sigma \mathrm{Gji}}{\mathrm{A}} \\
& \text { DORi }=\left(\frac{\text { DOAi }}{\Sigma \mathrm{DOA}}\right) * 100
\end{aligned}
$$

onde: $\mathrm{Gj}=$ Área Basal do indivíduo $\mathrm{j}\left(\mathrm{cm}^{2}\right) ; \mathrm{Gji}=$ Área Basal dos indivíduos $\mathrm{j}$, pertencentes à espécie i $\left(\mathrm{cm}^{2}\right) ;$ DOAi $=$ Dominância Absoluta da espécie i 
$\left(\mathrm{cm}^{2} / \mathrm{ha}\right) ;$ DORi $=$ Dominância Relativa da espécie i (\%); DAPj = Diâmetro a Altura do Peito do indivíduo $\mathrm{j}(\mathrm{cm}) ; \mathrm{A}=$ Área total amostrada (hectares).

Ao considerar os fatores de frequência, densidade e dominância é possível encontrar o Índice de Valor de Importância (IVI), o qual informa a importância ecológica das espécies em termos de distribuição horizontal na área estudada. Este índice foi calculado por meio da Equação 8 (Cottam \& Curtis, 1956; Mueller-Dombois \& Ellenberg, 1974):

$\mathrm{IVIi}=\frac{\mathrm{FRi}+\mathrm{DRi}+\mathrm{DORi}}{3}$

onde: IVIi = Índice de Valor de Importância da espécie i (\%); FRi = Frequência Relativa da espécie i (\%); DRi = Densidade Relativa da espécie i (\%); DORi $=$ Dominância Relativa da espécie i $(\%)$.

Após o cálculo dessas variáveis, os resultados foram estruturados por meio de gráficos e tabelas, buscando apresentar as principais informações geradas por este estudo, garantindo conhecimento básico para ser aplicado na gestão e manejo da área.

\section{Resultados}

A Praça Chácara Dona Catarina apresenta 93 indivíduos (83 árvores e 10 palmeiras), sendo que quatro destes indivíduos não foram botanicamente identificados e, consequentemente, foram desconsiderados para os demais resultados do presente estudo. Os indivíduos restantes estão distribuídos dentro de 21 espécies, classificadas em 19 gêneros e nove famílias botânicas (Quadro 2).

Quadro 2. Identificação e caracterização dos indivíduos arbóreos e palmeiras presente na Praça Chácara Dona Catarina, em Cataguases-MG. Fonte: Dalton, Nunes \& Martini (2021).

\begin{tabular}{|c|c|c|c|c|c|c|c|}
\hline Nome Comum & Nome Científico & Família & $\mathbf{O}$ & $\mathbf{C}$ & $\mathbf{P F}$ & NI & $\mathrm{F}(\%)$ \\
\hline Sibipiruna & $\begin{array}{c}\text { Cenostigma pluviosum (DC.) E. } \\
\text { Gagnon \& G.P. Lewis }\end{array}$ & Fabaceae & NL & - & SD & 31 & 34,83 \\
\hline Ipê-rosa & $\begin{array}{l}\text { Handroanthus impetiginosus } \\
\text { (Mart. ex DC.) Mattos }\end{array}$ & Bignoniaceae & NL & - & $\mathrm{D}$ & 18 & 20,22 \\
\hline $\begin{array}{l}\text { Palmeira-de- } \\
\text { saia }\end{array}$ & Washingtonia robusta $\mathrm{H}$. Wendl. & Arecaceae & $\mathrm{E}$ & $\mathrm{F}$ & $\mathrm{P}$ & 8 & 8,99 \\
\hline Ipê-tabaco & $\begin{array}{l}\text { Handroanthus chrysotrichus } \\
\text { (Mart. ex DC.) Mattos }\end{array}$ & Bignoniaceae & $\mathrm{N}$ & - & $\mathrm{D}$ & 7 & 7,87 \\
\hline Mangueira & Mangifera indica $\mathrm{L}$. & Anacardiaceae & $\mathrm{E}$ & $\mathrm{I}, \mathrm{F}$ & $\mathrm{P}$ & 4 & 4,49 \\
\hline Jambeiro & Syzygium jambos (L.) Alston & Myrtaceae & $E$ & $\mathrm{I}, \mathrm{F}$ & $\mathrm{P}$ & 3 & 3,37 \\
\hline Albízia & Albizia lebbeck (L.) Benth. & Fabaceae & $\mathrm{E}$ & $\mathrm{I}$ & $\mathrm{D}$ & 2 & 2,25 \\
\hline $\begin{array}{l}\text { Ameixa-da- } \\
\text { mata }\end{array}$ & Eugenia candolleana DC. & Myrtaceae & $\mathrm{N}^{*}$ & $\mathrm{~F}$ & $\mathrm{P}$ & 2 & 2,25 \\
\hline $\begin{array}{l}\text { Palmeira- } \\
\text { imperial }\end{array}$ & $\begin{array}{l}\text { Roystonea oleracea (Jacq.) O.F. } \\
\text { Cook }\end{array}$ & Arecaceae & $\mathrm{E}$ & $\mathrm{I}, \mathrm{F}$ & $\mathrm{P}$ & 2 & 2,25 \\
\hline Acácia-bastarda & Robinia pseudoacacia $\mathrm{L}$ & Fabaceae & $\mathrm{E}$ & I & $\mathrm{D}$ & 1 & 1,12 \\
\hline Aceroleira & Malpighia glabra L. & Malpighiaceae & $E$ & $\mathrm{~F}$ & $\mathrm{D}$ & 1 & 1,12 \\
\hline $\begin{array}{l}\text { Aroeira- } \\
\text { pimenta }\end{array}$ & Schinus terebinthifolia Raddi & Anacardiaceae & NL & - & $\mathrm{P}$ & 1 & 1,12 \\
\hline Caquizeiro & Diospyros kaki L.f. & Ebenaceae & $\mathrm{E}$ & $\mathrm{F}$ & $\mathrm{D}$ & 1 & 1,12 \\
\hline Caramboleira & Averrhoa carambola $\mathrm{L}$. & Oxalidaceae & $\mathrm{E}$ & $\mathrm{F}$ & $\mathrm{P}$ & 1 & 1,12 \\
\hline Figueira & Ficus benjamina L. & Moraceae & $\mathrm{E}$ & - & $\mathrm{P}$ & 1 & 1,12 \\
\hline Flamboyant & $\begin{array}{c}\text { Delonix regia (Bojer ex Hook.) } \\
\text { Raf. }\end{array}$ & Fabaceae & $\mathrm{E}$ & - & SD & 1 & 1,12 \\
\hline Goiabeira & Psidium guajava $\mathrm{L}$. & $\mathrm{N}$ & $\mathrm{N}$ & $\mathrm{I}, \mathrm{F}$ & $\mathrm{SD}$ & 1 & 1,12 \\
\hline Ipê-roxo & $\begin{array}{l}\text { Handroanthus heptaphyllus } \\
\text { (Vell.) Mattos }\end{array}$ & Bignoniaceae & $\mathrm{NL}$ & - & $\mathrm{D}$ & 1 & 1,12 \\
\hline Pau-brasil & $\begin{array}{c}\text { Paubrasilia echinata (Lam.) } \\
\text { Gagnon, H.C.Lima \& G.P. Lewis }\end{array}$ & Fabaceae & $\mathrm{NL}^{*}$ & - & SD & 1 & 1,12 \\
\hline Pau-ferro & $\begin{array}{l}\text { Libidibia ferrea (Mart. ex Tul.) } \\
\text { L.P. Queiroz }\end{array}$ & Fabaceae & $\mathrm{NL}^{*}$ & - & SD & 1 & 1,12 \\
\hline Tamarindeiro & Tamarindus indica $\mathrm{L}$. & Fabaceae & $\mathrm{E}$ & $\mathrm{F}$ & $\mathrm{P}$ & 1 & 1,12 \\
\hline \multicolumn{6}{|l|}{ Total } & 89 & 100 \\
\hline
\end{tabular}


nota: $\mathrm{O}=$ origem $(\mathrm{E}=$ exótica, $\mathrm{N}=$ nativa do Brasil e $\mathrm{NL}=$ nativa do local); $\mathrm{C}=$ características $(\mathrm{I}=$ espécie invasora e $\mathrm{F}=$ frutífera $) ; \mathrm{PF}=$ persistência das folhas $(\mathrm{P}=$ perenifólia, $\mathrm{SD}=$ semidecídua e $\mathrm{D}=$ decídua $)$; NI = número de indivíduos encontrados na praça; $\mathrm{F}=$ frequência da espécie em porcentagem; $*$ = endêmica do Brasil.

É possível observar que as famílias mais representadas, em relação ao número de indivíduos, foram: Fabaceae $(42,70 \%)$, Bignoneacea $(29,21 \%)$, Arecaceae $(11,24 \%)$, Myrtaceae (6,74\%) e Anacardiaceae (5,62\%). Ebenaceae, Malpighiaceae, Moraceae e Oxalidaceae apresentaram apenas um indivíduo em cada família.

Quanto à porcentagem de indivíduos distribuídos em cada família, foi possível observar que apenas Fabaceae ultrapassou a recomendação de não ter mais do que $30 \%$ de indivíduos de uma mesma família, e as espécies Cenostigma pluviosum e Handroanthus impetiginosus apresentaram mais de $10 \%$ do número de indivíduos. Juntas, essas duas espécies representam $55 \%$ dos indivíduos existentes.

Além disso, a análise da origem botânica indicou que a maioria das espécies são exóticas $(57,1 \%)$, seguidas pelas espécies nativas da Floresta Estacional Semidecidual $(28,6 \%)$ e nativas do Brasil (14,3\%). Porém, em número de indivíduos, as espécies da flora brasileira (nativas locais + nativas do Brasil), predominaram, representando 70,8\% dos exemplares. Assim, foram encontrados $59,6 \%$ de indivíduos classificados como nativos da Floresta Estacional Semidecidual, $11,2 \%$ nativos de outros ecossistemas brasileiros e 29,2\% de exóticos.

Foram, ainda, encontradas na Praça Chácara Dona Catarina, seis espécies consideradas invasoras, que juntas representam $14,6 \%$ do total de indivíduos, Mangifera indica, Syzygium jambos, Albizia lebbeck, Roystonea oleracea, Robinia pseudoacacia e Psidium guajava.

Entre todas as espécies analisadas, algumas podem ser consideradas como alimentícias para fauna silvestre e para a população humana, assim denominadas de frutíferas. No total, $47,6 \%$ das espécies da praça foram consideradas frutíferas, representadas por 24 exemplares (Figura 3).

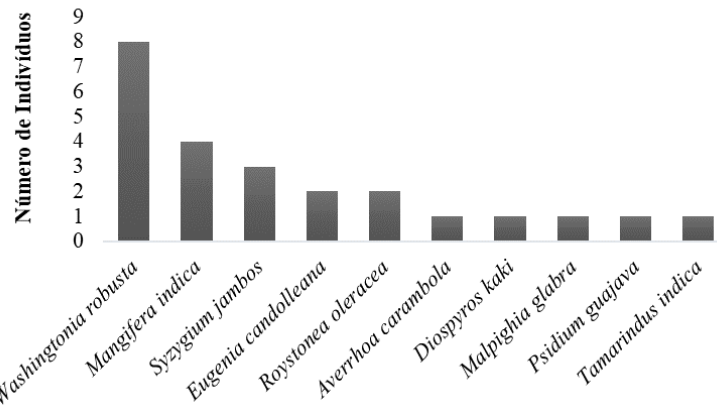

Figura 3. Espécies frutíferas separadas por número de indivíduos, encontradas na Praça Chácara Dona Catarina, em Cataguases-MG. Fonte: Dalton, Nunes \& Martini (2021).

Ao analisar a persistência foliar das espécies do local, verificou-se que a maioria apresenta copa perene $(42,8 \%)$, seguida pelas espécies de copa decídua $(33,3 \%)$ e semidecídua $(23,8 \%)$. No entanto, ao analisar a proporção de indivíduos, foi possível observar outra realidade, apenas $25,8 \%$ do total de árvores e palmeiras presentes na praça apresentam copa perene, $34,8 \%$ têm copa decídua e 39,3\% copa semidecídua.

Durante a avaliação das árvores quanto a qualidade do sombreamento gerado pelas mesmas, foi verificado que $75,3 \%$ dos indivíduos possuem as folhas bem distribuídas pela copa, de forma que promovam sombras adequadas no local em que se encontram. Em contrapartida, somente 2,2\% das árvores e palmeiras apresentam uma copa de qualidade baixa, com pouca ou nenhuma folhagem.

Em relação a distribuição dos indivíduos em classes de diâmetro, constatou-se que a maioria $(56,2 \%)$ possuía valores de DAP menores que 25 $\mathrm{cm}$, e que apenas três indivíduos apresentaram DAP maiores que $75 \mathrm{~cm}$. O modelo de distribuição "J-invertido", ou exponencial negativa, sugere que a população que compõem a praça apresenta uma distribuição diamétrica decrescente, indicando o predomínio de árvores e palmeiras com classes de DAP que representam dimensões de pequeno e médio porte (Figura 4). 


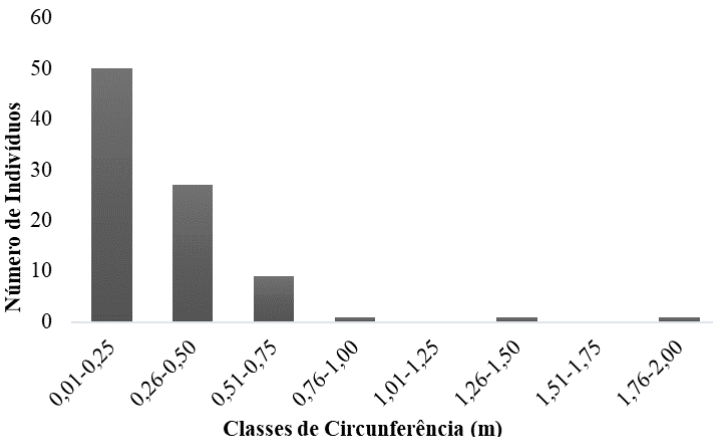

Figura 4. Distribuição diamétrica das árvores e palmeiras presentes na Praça Chácara Dona Catarina, em Cataguases-MG. Fonte: Dalton, Nunes \& Martini (2021).
Ao unir as informações dos alvos potenciais e do estado fitossanitário dos indivíduos, pode-se prosseguir com as análises de Índice de Risco, realizadas somente nos indivíduos que foram botanicamente identificados. Desta forma, foi possível verificar que $38,2 \%$ dos indivíduos apresentam baixo índice de risco, $52,8 \%$ foram categorizados como índice de risco médio e, por fim, 9,0\% dos indivíduos apresentam risco alto e, portanto, precisam ser monitorados de seis em seis meses (Figura 5). Esse monitoramento nos exemplares com risco alto é fundamental para garantir a segurança dos usuários da praça, bem como promover melhores condições para as árvores, por meio do manejo adequado.

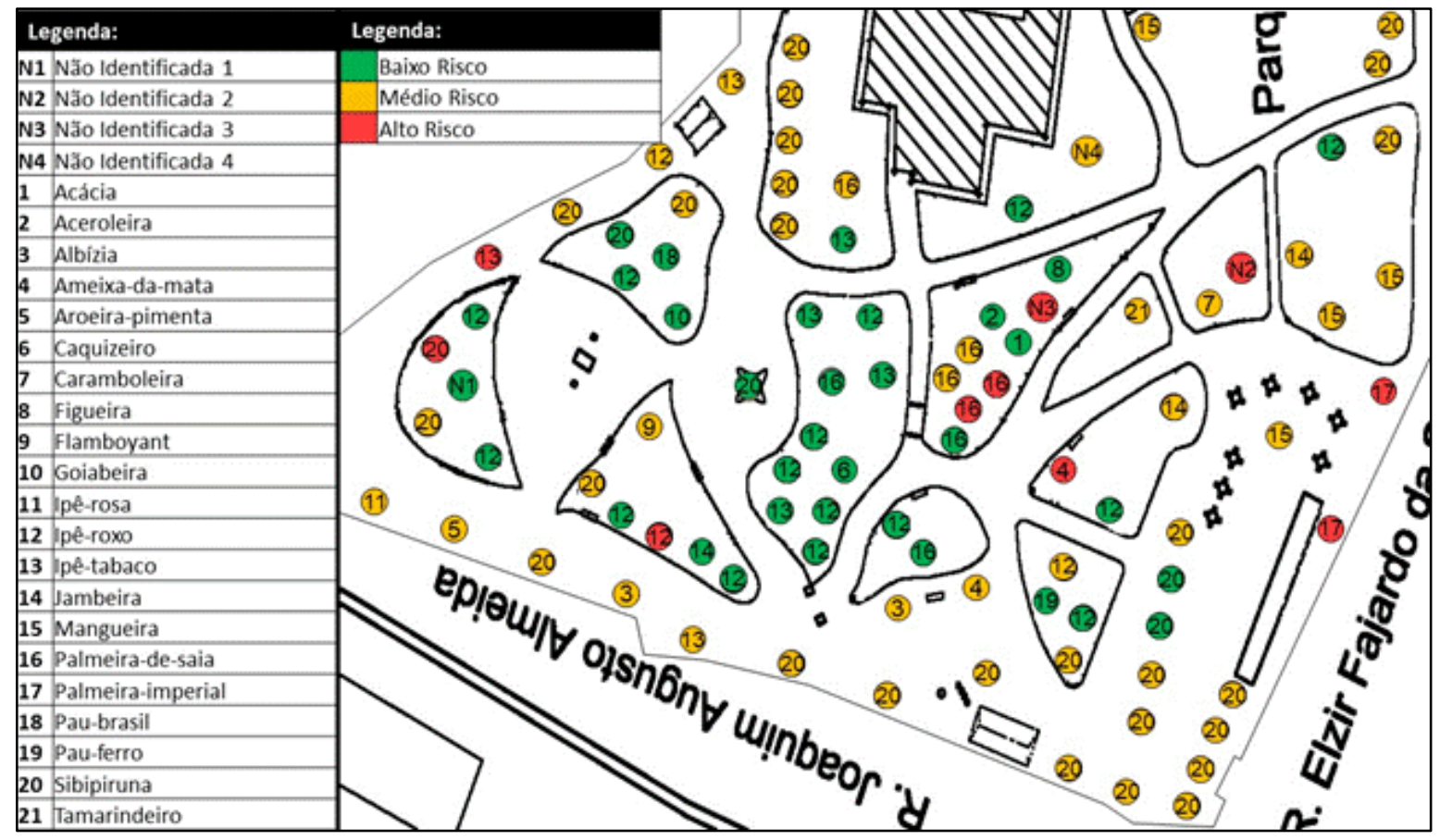

Figura 5. Croqui de distribuição das árvores e palmeiras com seus respectivos índices de risco na Praça Chácara Dona Catarina, em Cataguases-MG. Fonte: Dalton, Nunes \& Martini (2021).

Os indivíduos classificados em alto risco referem-se as árvores e palmeiras que, além de estarem localizados em áreas com alvo potencial de alto valor econômico ou de intenso fluxo de pessoas, apresentam problemas fitossanitários, em sua maioria, como lesões nos troncos, como ocos e rachaduras. Aqueles classificados com o índice de risco em valor médio, podem tanto estar saudáveis, mas localizados próximos de calçadas, pontos de ônibus ou outros pontos com um alvo potencial categorizado como importante, ou podem estar em locais sem grandes riscos de danos em caso de queda, mas com alguns problemas fitossanitários mais graves. Por fim, os indivíduos de baixo risco não apresentaram perigo de atingir um alvo potencial de grande importância, nem sérios problemas fitossanitários (Figura 6).

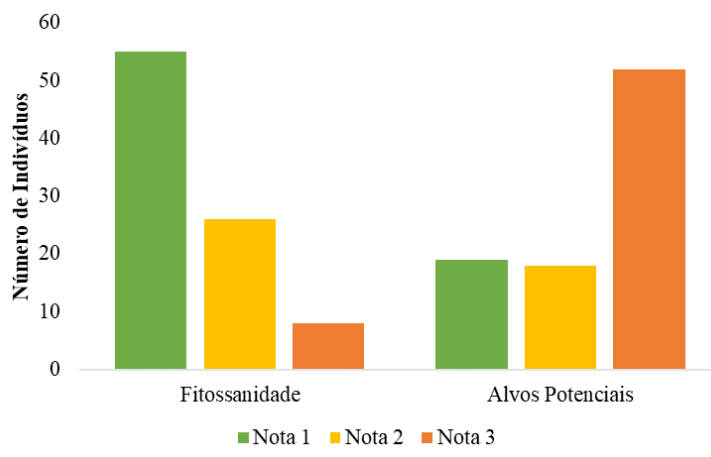

Figura 6. Avaliações de fitossanidade e de alvos potenciais das árvores e palmeiras presentes na Praça Chácara Dona Catarina, em Cataguases-MG. Fonte: Dalton, Nunes \& Martini (2021). 
Quanto à análise da substituibilidade, em caso de necessidade de remoção imediata dos indivíduos, constatou-se que $67,4 \%$ não apresentariam grandes dificuldades de serem replantados, pois localizam-se em grandes canteiros, com espaço amplo o suficiente para que o indivíduo possa ser substituído. No entanto, os $32,6 \%$ restantes dos indivíduos estão localizados em canteiros restritos distribuídos pela praça, sendo que 23,6\% foram categorizados com um nível médio de dificuldade para a substituibilidade, e somente $9,0 \%$ dificilmente poderão ser substituídos devido à falta de espaço no canteiro em que se encontram.

Por fim, os parâmetros fitossociológicos calculados para a vegetação da Praça Chácara Dona Catarina, indicam que a espécie com maior densidade relativa foi a Cenostigma pluviosum (Figura 7) e a de maior dominância relativa foi Mangifera indica (Figura 8). Destaca-se ainda, que a área da praça mensurada para a realização desses cálculos foi de 0,6323 ha.

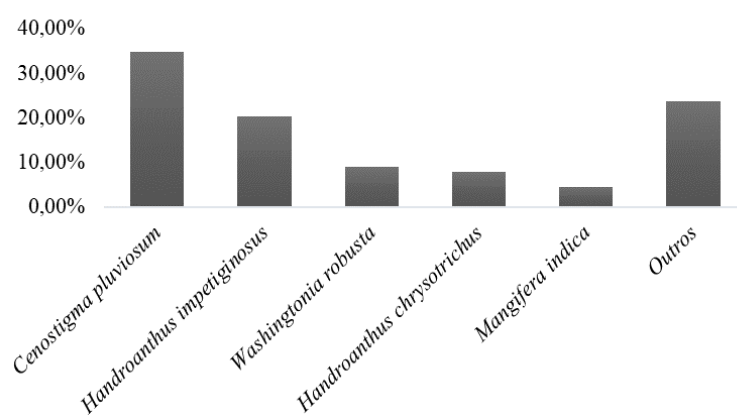

Figura 7. Densidade relativa relativa das espécies de árvores e palmeiras presentes na Praça Chácara Dona Catarina, em Cataguases-MG. Fonte: Dalton, Nunes \& Martini (2021).

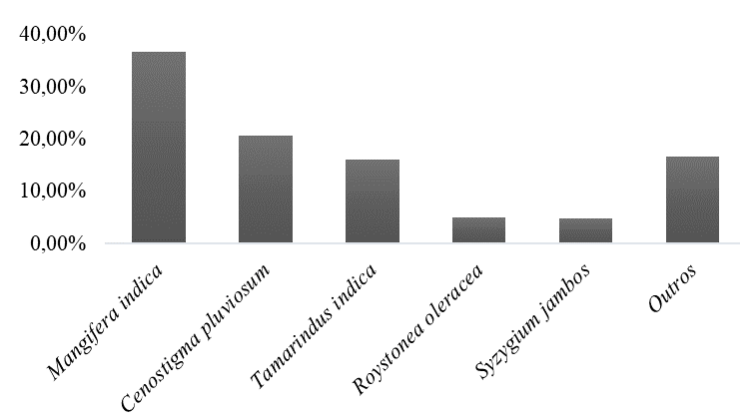

Figura 8. Dominância relativa relativa das espécies de árvores e palmeiras presentes na Praça Chácara Dona Catarina, em Cataguases-MG. Fonte: Dalton, Nunes \& Martini (2021).

O maior número de indivíduos de Cenostigma pluviosum fez com que esta espécie apresentasse a maior densidade relativa da praça, enquanto, para a dominância relativa, foi possível observar que esse parâmetro recebeu maior influência da variável CAP do que do número de indivíduos, isto porque Mangifera indica foi considerada a espécie mais dominante. Vale ressaltar que outras 16 espécies compõe a categoria denominada outros, com valores individuais inferiores a $2,25 \%$, e que com os devidos cálculos realizados, foi possível determinar o índice de Valor de importância para cada espécie (Figura 9).

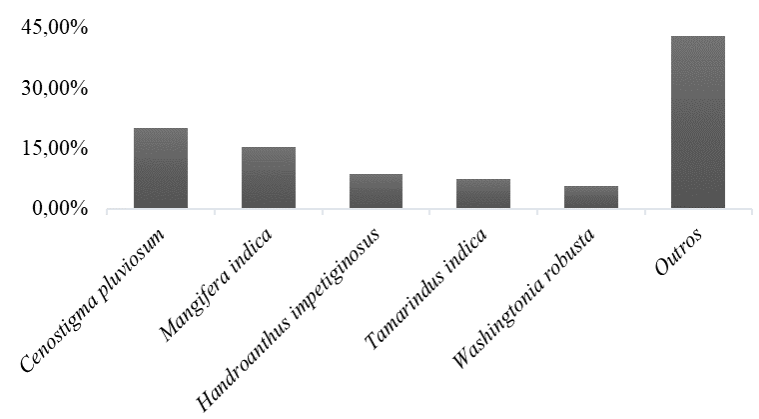

Figura 9. Índice de Valor de Importância das espécies de árvores e palmeiras presentes na Praça Chácara Dona Catarina, em Cataguases-MG. Fonte: Dalton, Nunes \& Martini (2021).

Dentre as 21 espécies analisadas, pode-se afirmar que as cinco mais importantes em termos fitossociológicos para a praça foram, nesta ordem: Cenostigma pluviosum, Mangifera indica, Handroanthus impetiginosus, Tamarindus indica $\mathrm{e}$ Washingtonia robusta. Tais espécies, seja pela alta densidade de seus exemplares, como no caso de Cenostigma pluviosum e Handroanthus impetiginosus, ou seja, pelas grandes dimensões de DAP, como ocorre com os indivíduos de Mangifera indica e Tamarindus indica, são as mais importantes para a praça, e por isso devem receber maior atenção por parte da gestão municipal.

\section{Discussão}

O maior número de indivíduos pertencentes a Fabaceae é evidenciado corriqueiramente na arborização de praças urbanas do Brasil (Lindenmaier \& Santos, 2008). Em estudo semelhante, realizado na Praça Matriz em Godoy Moreira, no Paraná, 50\% dos indivíduos pertenciam a esta família e a segunda família mais frequente, assim como na presente pesquisa, também foi Bignoniaceae, com 28,56\% dos indivíduos (Miranda, Larocca \& Angelis, 2017).

Fabaceae apresentou porcentagem alta quanto ao número de indivíduos, o que contraria indicação de Donamour (2002), que não recomenda que uma área urbana tenha mais de $10 \%$ de indivíduos de uma mesma espécie. Nesta praça constatou-se indivíduos acima de $10 \%$ para as 
espécies Cenostigma pluviosum e Handroanthus impetiginosus. Outros autores advertem que para uma arborização adequada, é desejável que o número de indivíduos de uma espécie não ultrapasse $15 \%$ do total de indivíduos da população arbórea (Milano \& Dalcin, 2000). Essa heterogeneidade de espécies e famílias é importante para diluir os riscos com a arborização, evitando que todo o ambiente seja dizimado por um surto de pragas e doenças (Albertin et al., 2011).

Embora Cenostigma pluviosum e

Handroanthus impetiginosus ocorram em proporções maiores do que o recomendado, ambas espécies são as principais responsáveis pelo baixo índice de indivíduos exóticos encontrados na praça, pois mesmo que o número de espécies exóticas seja maior do que o de espécies nativas, a quantidade de indivíduos de origem de fora do Brasil foi menor. Este resultado foi exatamente o contrário do encontrado na Praça Nossa Senhora da Conceição, em Macapá - AP, onde o número de espécies nativas em relação as exóticas foi maior, mas a quantidade de indivíduos de origem exótica na composição do paisagismo da praça foi expressivamente maior (Gomes et al., 2016).

Vale ressaltar que presença de espécies nativas tem grande importância no meio urbano, desde a preservação da ornamentação original, até a atração da avifauna local, sendo que as espécies nativas estão naturalmente adaptadas às condições edafoclimáticas de sua área de origem (Dias \& Costa, 2008; Santos, Silva \& Souza, 2011). Desta forma, destaca-se que exatamente 8 das 10 espécies classificadas como frutíferas na praça são de origem exótica, um número elevado. Além disso, três destas espécies são potenciais invasoras no território brasileiro, Mangifera indica, Syzygium jambos e Roystonea oleracea. É importante também mencionar a Psidium guajava, outra espécie frutífera com potencial de invasão, mas que é considerada naturalizada no Brasil.

A maior parte das árvores da praça são plantas jovens que estão enquadradas na classe de diâmetro que varia de 1 a 25 centímetros, o que significa que, no futuro, há de se haver uma melhor qualidade de sombreamento, além de uma maior diversificação na paisagem vegetal, visto que estes 50 indivíduos recém-plantados são representados, em sua maioria, por espécies pouco frequentes na amostra. A predominância de árvores em classes de menores DAP é típica da distribuição diamétrica decrescente, que, de acordo com Souza et al. (2006), indica uma tendência futura de distribuição balanceada, como ocorre na Praça República do Iraque, em Belo Horizonte-MG, onde a distribuição das classes de diâmetro apresenta uma prevalência de árvores de médio e grande porte, formando uma curva de distribuição unimodal (Silva \& Ataíde, 2019).

O amplo espaçamento nos canteiros e a ausência de rede elétrica aérea no local, permitem o uso de espécies de grande porte na praça, contribuindo para intensificar o sombreamento de regiões importantes, como nas diversas mesas de jogos e nos pontos de ônibus, locais que tendem a ter um fluxo elevado de pessoas. Além disso, um espaço de canteiro adequado é fundamental para que haja o crescimento e desenvolvimento das árvores, além de conferir a permeabilidade de água no solo (Gonçalves \& Paiva, 2013).

Apesar de apenas dois indivíduos apresentarem copas de qualidades ruins, principalmente por motivos fitossanitários que causam desfolha, a maioria das árvores e palmeiras apresentam copas decíduas e semidecíduas, o que interfere na intensidade de sombra em determinados períodos do ano. Aproximadamente um quarto dos indivíduos possuem copa perene, onde a permanência de folhas ocorre ao longo de todo o ano. Este valor é bem abaixo do encontrado na Praça Miguel Rossafa, em Umuarama - PR, cuja porcentagem de indivíduos de copa perene foi de 57\% (Cardoso, Miranda \& Angelis, 2017).

A maior parcela das árvores e palmeiras apresentaram boas condições fitossanitárias, tanto nas folhas quanto nas partes estruturais, como raízes, troncos e galhos. Portanto, o fato de que a qualificação de Índice de Risco médio tenha sido a mais comum para a maior parte dos indivíduos, pode ser explicado pela classificação dos alvos potenciais, visto que, em um caso de queda, a maioria das árvores poderiam atingir pessoas, carros e outras estruturas presentes na praça. Somente indivíduos de pequeno porte, localizados nos interiores de grandes canteiros, mostraram um baixo risco de atingir um alvo potencial. Ainda assim, é indicado que sejam avaliados todos os indivíduos, independentemente de seu Índice de Risco, sempre após eventos ambientais extremos, como tempestades.

Analisando os parâmetros fitossociológicos que compõem o Índice de Valor de Importância (IVI) é possível analisar que a Cenostigma pluviosum, além de ser a espécie com maior densidade na amostra, é também a mais importante do local, diferentemente da Praça XV de Novembro, em Ribeirão Preto, São Paulo, onde Romani (2011) destacou que a Cenostigma pluviosum, mesmo possuindo a maior densidade relativa do local, não foi a espécie com maior Índice de Valor de Importância, devido à baixa dominância relativa dos indivíduos desta espécie. É importante ressaltar que, no presente estudo, os valores de frequência absoluta e relativa das 
espécies foram os mesmos, visto que somente uma unidade amostral foi analisada.

A demonstração de que a dominância também possui influência nos valores de IVI pode ser observada no exemplo da Mangifera indica, espécie de maior dominância e segunda mais importante da praça, sendo somente a quinta espécie com maior densidade de indivíduos no local.

\section{Conclusão}

A praça da Chácara Dona Catarina apresentou uma variedade de 21 espécies de porte arbóreo, com mais da metade dos indivíduos pertencentes as espécies Cenostigma pluviosum e Handroanthus impetiginosus. Além disso, o conjunto de diferentes árvores e palmeiras de origens geográficas distintas contribui para um paisagismo com heterogeneidade de formas, tamanhos, cores e texturas no local.

Por fim, para tomadas de decisão mais assertivas sobre o manejo e o planejamento da arborização do local, é importante considerar os fatores levantados neste estudo, como a origem botânica, o potencial de invasão das espécies, a persistência foliar, os valores de importância calculados e a periodicidade de avaliação dos indivíduos, proposta com base no Índice de Risco. Recomenda-se que, posteriormente, um estudo semelhante seja conduzido no mesmo local, para comparação e atualização das necessidades de manejo.

\section{Agradecimentos}

Os autores agradecem ao Departamento de Engenharia Florestal e à Secretaria de Agricultura e Meio Ambiente da Prefeitura Municipal de Cataguases pela contribuição e apoio na elaboração deste estudo.

\section{Referências}

Abreu, R. L. de. 2020. Mapa de localização da cidade de Cataguases, Minas Gerais. Disponível em: https://commons.wikimedia.org/wiki/File:Mi nasGerais_Municip_Cataguases.svg. Acesso em: 19 de fevereiro de 2020.

Albertin, R. M.; Angelis, R. de.; Angelis, G. N.; Angelis, B. L. D. de. 2011. Diagnóstico Quali-Quantitativo da Arborização Viária de Nova Esperança, Paraná, Brasil. Revista da Sociedade Brasileira de Arborização Urbana, 6, 3, 128-148. http://dx.doi.org/10.5380/ revsbau.v6i3.66477

Alonso, P. H. 2012a. Guia da Arquitetura Modernista de Cataguases. Instituto Cidade de Cataguases, Segunda Edição.
Alonso, P. H. 2012b. Memória e Patrimônio Cultural de Cataguases. Instituto Cidade de Cataguases, Segunda Edição.

Aragão, L. A. 2016. Peso e Composição da Serapilheira em Reflorestamentos de Diferentes Idades. Monografia de Bacharelado, Universidade Federal Rural do Rio de Janeiro. Três Rios, Rio de Janeiro, Brasil. 38p.

Benini, S. M.; Martin, E. S. 2010. Decifrando áreas verdes públicas. Revista Formação, 2, 17, 63 80. https://doi.org/10.33081/formacao. v2i 17.455

Cardoso, C.; Miranda, Y. C.; Angelis, B. L. D. 2017. Análise da Requalificação da Praça Miguel Rossafa, Umuarama-PR. Revista Brasileira de Gerenciamento de Cidades, 5, 29, 126-139. http://dx.doi.org/10.17271/231 8847252920171525

Cottam, G.; Curtis, J. T. 1956. The use of distance measures in phytosociological sampling. Ecology, 37, 3, 451-460. https://doi.org/10.23 07/1930167

Dias, J.; Costa, L. D. 2008. Sugestões de Espécies Arbóreas Nativas Ocorrentes no Sul do Estado do Paraná para Fins Ornamentais. $8^{\circ}$ Encontro de Iniciação Científica, pp. 1-8.

Gomes, E. M. C.; Rodrigues, D. M. de S.; Santos, J. T.; Barbosa, E. de J. 2016. Análise qualiquantitativa da arborização de uma praça urbana do norte do Brasil. Nativa, 4, 3, 179186. https://doi.org/10.14583/2318-7670. V04N03A12

Gonçalves, W.; Paiva, H. N. 2013. Implantação da arborização urbana: especificações técnicas. Editora UFV, Primeira Edição.

Hubacek, K.; Kronenberg, J. 2013. Synthesizing different perspectives on the value of urban ecosystem services. Landscape and Urban Planning, 109, 1, 1-6. https://doi.org/10.1016/ j.landurbplan.2012.10.010

IBGE. Área Territorial Oficial. Disponível em: https://www.ibge.gov.br/. Acesso em: 19 de fevereiro de 2020.

Jardim Botânico do Rio de Janeiro. Flora do Brasil $2020 . \quad$ Disponível em: http://reflora.jbrj.gov.br/reflora/listaBrasil/C onsultaPublicaUC/ResultadoDaConsultaNov aConsulta.do\#CondicaoTaxonCP. Acesso em: 19 de fevereiro de 2020.

Kottek, M.; Grieser, J.; Beck, C.; Rudolf, B.; Rubel, F. 2006. World Map of the KöppenGeiger climate classification updated. Meteorologische Zeitschrift, 15, 3, 259-263. https://doi.org/10.1127/0941-2948/2006/01 30 
Lindenmaier, D. D. S.; Santos, N. O. 2008. Arborização urbana das praças de Cachoeira do Sul, Rio Grande do Sul, Brasil: Fitogeografia, Diversidade e Resumo. Pesquisas, Botânica, 1, 59, 307-319.

Melo, E. F. R. Q.; Romanini, A. 2008. Praça Ernesto Tochetto: Importância da sua preservação histórica e aspectos de sua arborização. Revista da Sociedade Brasileira de Arborização Urbana, 3, 1, 54-72. http://dx.doi.org/10.5380/revsbau.v3i1.6625 1

Milano, M.; Dalcin, E. 2000. Arborização de vias públicas. Light: Rio de Janeiro, Primeira Edição.

Miranda, Y. C.; Larocca, A. G.; Angelis, B. L. D. 2017. Análise quali-quantitativa da Arborização na Praça da Matriz, em Godoy Moreira-PR. Revista Nacional de Gerenciamento de Cidades, 5, 36, 80-90. http://dx.doi.org/10.17271/23188472536201 71635

Mueller-Dombois, D.; Ellenberg, H. 1974. Aims and methods of vegetation ecology. New York: Wiley \& Sons, 547p. http://dx.doi.org/10.2307/213332

Nielsen, A. B.; Östberg, J.; Delshammar, T. 2014. Review of Urban Tree Inventory Methods Used to Collect Data at Single-Tree Level. Arboriculture \& Urban Forestry, 40, 2, 96111. http://dx.doi.org/10.48044/jauf.2014. 011

Oliveira, A. S. de.; Sanches, L.; Musis, C. R. de.; Nogueira, M. C. de J. A. 2013. Benefícios da arborização em praças urbanas - $\mathrm{O}$ caso de Cuiabá / MT. Revista Eletrônica em Gestão, Educação e Tecnologia Ambiental, 9, 9, 1900-1915. http://dx.doi.org/10.5902/223611 707695

Paula, L. de.; Duarte, M. S. S.; Tostes, R. B.; Ruback, S. de S. 2015. Arborização urbana do bairro Centro do município de Cataguases, MG. Revista Agrogeoambiental, 7, 2, 101112. http://dx.doi.org/10.18406/2316$1817 \mathrm{v} 7 \mathrm{n} 22015708$

Rodrigues, C. F. A. 2019. Fitossociologia e análise temporal do fragmento florestal urbano Capoeira do Black. Dissertação de Mestrado, Universidade Federal Rural da Amazônia. Belém, Pará, Brasil. 84p.

Romani, G. de N. 2011. Análise florística fitossociológica e qualitativa da arborização na Praça XV de Novembro em Ribeirão Preto, SP. Dissertação de Mestrado, UNESP. Jaboticabal, São Paulo, Brasil. 73p.

Romani, G. de N.; Gimenes, R.; Silva, M. T.; Pivetta, K. F. L.; Batista, G. S. 2012. Análise quali-quantitativa da arborização na praça XV de Novembro em Ribeirão Preto - SP, Brasil. Revista Árvore, 36, 3, 479-487. https://doi. org/10.1590/S0100-67622012000300010

Santamour, F. S. 2002. Trees for urban planting: Diversity, Uniformity, and Common Sense. US National Arboretum, 7, 1, 57-65.

Santana, C. A. de A.; Silva, V. G. da; Silva, A. T. 2016. Manual de Identificação de Mudas de Espécies Florestais. Secretaria Municipal de Meio Ambiente, Rio de Janeiro, Segunda Edição.

Santos, A. C. B. dos; Silva, M. A. P. da; Souza, R. K. D. 2011. Levantamento Florístico das Espécies Utilizadas na Arborização de Praças do Município de Crato, CE. Caderno de Cultura e Ciência, 10, 1, 13-18.

Santos, C. R. dos; Lage, C. F. 2005. Cataguases: Patrimônio da Modernidade. Disponível em: https://www.vitruvius.com.br/revistas/read/a rquitextos/05.056/512. Acesso em: 19 de fevereiro de 2020.

Santos, C. Z. dos.; Ferreira, R. A.; Santos, L. R.; Santos, L. I.; Graça, D. A. S. da; Gomes, S. H.; Porto Neto, W. de B.; Correia, T. S.; Boschese, A. C. de B. 2011. Composição florística de 25 vias públicas de Aracaju - SE. Revista da Sociedade Brasileira de Arborização Urbana, 6, 2, 125-144. http://dx.doi.org/10.5380/revsbau.v6i2.6640 7

Silva, A. D. P.; Batista, A. C.; Giongo, M. V.; Biondi, D.; Santos, A. F. dos. Oliveira, L. M. de.; Cachoeira, J. N. 2019. Arborização das praças de Gurupi-TO-Brasil: Composição e diversidade de espécies. Revista da Sociedade Brasileira de Arborização Urbana, 14, 4, 112 . http://dx.doi.org/10.5380/rev sbau.v14i4.67547

Silva, A. G. da.; Ataíde, G. da M. 2019. Inventário e Diagnóstico da Arborização da Praça Pública do Iraque em Belo Horizonte. Agrarian Academy, 6, 12, 61-69.

Simão, M. V. R. de C. et al. 2017. Árvores da Mata Atlântica. Editora UFV, $1^{a}$ edição.

Souza, D. R. de.; Souza, A. L. de.; Leite, H. G.; Yared, J. A. G. 2006. Análise estrutural em floresta ombrófila densa de terra firme não explorada, Amazônia oriental. Revista Arvore, 30, 1, 75-87. https://doi.org/ 10.1590/S0100-67622006000100010

Souza, V. C.; Lorenzi, H. 2007. Chave de Identificação: para as principais famílias de Angiospermas nativas e cultivadas do Brasil. Instituto Platarum, $2^{\mathrm{a}}$ Edição. 